\title{
A Measurable-Group-Theoretic Solution to von Neumann's Problem
}

\author{
Damien Gaboriau* Russell Lyons ${ }^{\dagger}$
}

October 23,2018

\begin{abstract}
We give a positive answer, in the measurable-group-theory context, to von Neumann's problem of knowing whether a non-amenable countable discrete group contains a non-cyclic free subgroup. We also get an embedding result of the free-group von Neumann factor into restricted wreath product factors.
\end{abstract}

2000 Mathematical Subject Classification: 37A20, 20E05, 82B43, 05C80

Key words and phrases: Orbit equivalence, free group, non-amenability, von Neumann's problem, percolation theory, graphs, trees

Amenability of groups is a concept introduced by J. von Neumann in his seminal article vN29] to explain the so-called Banach-Tarski paradox. He proved that a discrete group containing the free group $\mathbf{F}_{2}$ on two generators as a subgroup is non-amenable. Knowing whether this was a characterization of non-amenability became known as von Neumann's Problem and was solved by the negative by A. Ol'šanskiı [ $\mathrm{Ol}^{\prime} 80$. Still, this characterization could become true after relaxing the notion of "containing a subgroup". K. Whyte gave a very satisfying geometric group-theoretic solution: A finitely generated group $\Gamma$ is non-amenable iff it admits a partition with pieces uniformly bilipschitz equivalent to the regular 4-valent tree [Why99]. Geometric group theory admits a measurable counterpart, namely, measurable group theory. The main goal of our note is to provide a solution to von Neumann's problem in this context. We show that any countable non-amenable group admits a measure-preserving free action on some probability space, such that the orbits may be measurably partitioned into pieces given by an $\mathbf{F}_{2}$-action.

To be more precise, we use the following notation. For a finite or countable set $M$, let $\mu$ denote the product $\otimes_{M} L e b$ on $[0,1]^{M}$ of the Lebesgue measures $L e b$ on $[0,1]$, and for $p \in[0,1]$, let $\mu_{p}$ denote the product of the discrete measures $(1-p) \delta_{\{0\}}+p \delta_{\{1\}}$ on $\{0,1\}^{M}$. Thus, the meaning of $\mu$ may vary from use to use as $M$ varies. Usually $M$ will be a countable group $\Lambda$ or the set $\mathrm{E}$ of edges of a Cayley graph.

\section{${ }^{*} \mathrm{CNRS}$}

${ }^{\dagger}$ Supported partially by NSF grant DMS-0705518 and Microsoft Research. 
Theorem 1 For any countable discrete non-amenable group $\Lambda$, there is a measurable ergodic essentially free action of $\mathbf{F}_{2}$ on $\left([0,1]^{\Lambda}, \mu\right)$ such that almost every $\Lambda$-orbit of the Bernoulli shift decomposes into $\mathbf{F}_{2}$-orbits.

In other words, the orbit equivalence relation of the $\mathbf{F}_{2}$-action is contained in that of the $\Lambda$-action. We give two proofs of this theorem, each with its own advantages.

For some purposes, it is useful to get a Bernoulli shift action with a discrete base space. We show:

Theorem 2 For any finitely generated non-amenable group $\Gamma$, there is $n \in \mathbb{N}$ and a non-empty interval $\left(p_{1}, p_{2}\right)$ of parameters $p$ for which there is an ergodic essentially free action of $\mathbf{F}_{2}$ on $\prod_{1}^{n}\left(\{0,1\}^{\Gamma}, \mu_{p}\right)$ such that almost every $\Gamma$-orbit of the diagonal Bernoulli shift decomposes into $\mathbf{F}_{2}$-orbits.

These results have operator-algebra counterparts:

Corollary 3 Let $\Lambda$ be a countable discrete non-amenable group and $H$ be an infinite group. Then the von Neumann factor $L(H<\Lambda)$ of the restricted wreath product contains a copy of the von Neumann factor $L\left(\mathbf{F}_{2}\right)$ of the free group.

Corollary 4 Let $\Gamma$ be a finitely generated discrete non-amenable group. Let $n, p_{1}, p_{2}$ be as in Theorem 2 and let $p=\frac{\alpha}{\beta} \in\left(p_{1}, p_{2}\right)$, with $\alpha, \beta \in \mathbb{N}$. Assume that $H$ contains an abelian subgroup $K$ of order $k=\beta^{n}$. Then the von Neumann factor $L(H<\Lambda)$ of the restricted wreath product contains a copy of the von Neumann factor $L\left(\mathbf{F}_{2}\right)$ of the free group.

For this paper, we assume a certain familiarity with the results and notation of [Gab05], Gab00] and [LS99].

\section{Acknowledgment}

We are very grateful to Sorin Popa for bringing to our attention the above corollaries. We also thank the referee for a careful reading.

A (countable standard) equivalence relation on the standard Borel space $(X, \nu)$ is an equivalence relation $\mathcal{R}$ with countable classes that is a Borel subset of $X \times X$ for the product $\sigma$-algebra.

A (measure-preserving oriented) graphing on $(X, \nu)$ is a denumerable family $\Phi=\left(\varphi_{i}\right)_{i \in I}$ of partial measure-preserving isomorphisms $\varphi_{i}: A_{i} \rightarrow B_{i}$ between Borel subsets $A_{i}, B_{i} \subset X$.

A graphing $\Phi$ generates an equivalence relation $\mathcal{R}_{\Phi}$ : the smallest equivalence relation that contains all pairs $\left(x, \varphi_{i}(x)\right)$. The cost of a graphing $\Phi=\left(\varphi_{i}\right)_{i \in I}$ is the sum of the measures of the domains $\sum_{i \in I} \nu\left(A_{i}\right)$. The $\operatorname{cost}, \operatorname{cost}(\mathcal{R}, \nu)$, of $(\mathcal{R}, \nu)$ is the infimum of the costs of the graphings that generate $\mathcal{R}$. The graph (structure) $\Phi[x]$ of a graphing $\Phi$ at a point $x \in X$ is the graph whose vertex set is the equivalence class $\mathcal{R}_{\Phi}[x]$ of $x$ and whose edges are the pairs $(y, z) \in \mathcal{R}_{\Phi}[x] \times \mathcal{R}_{\Phi}[x]$ such that for some $i \in I$, either $\varphi_{i}(y)=z$ or $\varphi_{i}(z)=y$. For more on cost, see [Gab00] or [KM04]. 


\section{Proofs}

Since the union of an increasing sequence of amenable groups is still amenable, $\Lambda$ contains a non-amenable finitely generated subgroup. Let $\Gamma$ be such a subgroup.

If $S$ is a finite generating set of $\Gamma$ (maybe with repetitions), $\mathcal{G}=(\mathrm{V}, \mathrm{E})$ denotes the associated right Cayley graph (with vertex set V): The set $\mathrm{E}$ of edges is indexed by $S$ and $\Gamma$. Given $s \in S$ and $\gamma \in \Gamma$, the corresponding edge is oriented from the vertex $\gamma$ to $\gamma s$. Note that $\Gamma$ acts freely on $\mathcal{G}$ by multiplication on the left. Let $\rho:=$ id, the identity of the group $\Gamma$, chosen as base vertex for $\mathcal{G}$.

The set of the subgraphs of $\mathcal{G}$ (with the same set of vertices $\mathrm{V}$ ) is naturally identified with $\Omega:=$ $\{0,1\}^{\mathrm{E}}$. The connected components of $\omega \in \Omega$ are called its clusters.

Consider a probability-measure-preserving essentially free (left) $\Gamma$-action on some standard Borel space $(X, \nu)$ together with a $\Gamma$-equivariant Borel map $\pi: X \rightarrow\{0,1\}^{\mathrm{E}}$.

The full equivalence relation $\mathcal{R}_{\Gamma}$ generated by the $\Gamma$-action $X$ is graphed by the graphing $\Phi=$ $\left(\varphi_{s}\right)_{s \in S}$, where $\varphi_{s}$ denotes the action by $s^{-1}$.

We define the following equivalence subrelation on $X$ (see [Gab05, Sect. 1]): the cluster equivalence subrelation $\mathcal{R}^{\mathrm{cl}}$, graphed by the graphing $\Phi^{\mathrm{cl}}:=\left(\varphi_{s}^{\mathrm{cl}}\right)_{s \in S}$ of partial isomorphisms, where $\varphi_{s}^{\mathrm{cl}}$ is the restriction $\varphi_{s}^{\mathrm{cl}}:=\varphi_{s} \mid A_{s}$ of $\varphi_{s}$ to the Borel subset $A_{s}$ of $x \in X$ for which the edge $e$ labelled $s$ from $\rho$ to $\rho s$ lies in $\pi(x)$, i.e., $\pi(x)(e)=1$. Consequently, two points $x, y \in X$ are $\mathcal{R}^{\mathrm{cl}}$-equivalent if and only if there is some $\gamma \in \Gamma$ such that $\gamma^{-1} x=y$ and the vertices $\rho, \gamma \rho$ are in the same cluster of $\pi(x)$.

The graph structure $\Phi^{\mathrm{cl}}[x]$ given by the graphing $\Phi^{\mathrm{cl}}$ to the $\mathcal{R}^{\mathrm{cl}}$-class of any $x \in X$ is naturally isomorphic with the cluster $\pi(x)_{\rho}$ of $\pi(x)$ that contains the base vertex. Denote by $U^{\infty} \subset X$ the Borel set of $x \in X$ whose $\mathcal{R}^{\text {cl }}$-class is infinite and by $\mathcal{R}_{\Gamma \mid \infty}\left(\right.$ resp. $\left.\mathcal{R}_{\mid \infty}^{\text {cl }}\right)$ the restriction of $\mathcal{R}_{\Gamma}\left(\right.$ resp. $\left.\mathcal{R}^{\text {cl }}\right)$ to $U^{\infty}$.

Write $\mathcal{P}(Y)$ for the power set of $Y$. The map $X \times \mathrm{V} \rightarrow X$ defined by $(x, \gamma \rho) \mapsto \gamma^{-1} . x$ induces a map $\Psi: X \times \mathcal{P}(\mathrm{V}) \rightarrow \mathcal{P}(X)$ that is invariant under the (left) diagonal $\Gamma$-action (i.e., $\Psi(\gamma . x, \gamma \cdot C)=\Psi(x, C)$ for all $\gamma \in \Gamma, x \in X$, and $C \subset \mathrm{V})$ and such that $\Psi(x, \mathrm{~V})$ is the whole $\mathcal{R}_{\Gamma^{-}}$-class of $x$. The restriction of $\Psi$ to the $\Gamma$-invariant subset $\mathfrak{C}_{\infty}^{\mathrm{cl}}:=\{(x, C): x \in X, C \in \mathcal{P}(\mathrm{V}), C$ is an infinite cluster of $\pi(x)\}$ sends each $(x, C)$ (and its $\Gamma$-orbit) to a whole infinite $\mathcal{R}^{\mathrm{cl}}$-class, namely, the $\mathcal{R}^{\mathrm{cl}}$-class of $\gamma^{-1} \cdot x$ for any $\gamma$ such that $\gamma \rho \in C$. Moreover, for each $x \in U^{\infty}$, its $\mathcal{R}_{\Gamma \mid \infty}$-class decomposes into infinite $\mathcal{R}^{\text {cl }}$-sub-classes that are in one-to-one correspondence with the elements of $\mathfrak{C}_{\infty}^{\mathrm{cl}}$ that have $x$ as first coordinate. Note that the set $\{(x, y, C) \in X \times X \times \mathcal{P}(\mathrm{v}): x \in \Psi(y, C)\}$ is Borel, whence for a Borel set $\mathcal{A} \subset \mathfrak{C}_{\infty}^{\mathrm{cl}}$, the set $\bar{\Psi}(\mathcal{A}):=\bigcup \Psi(\mathcal{A})$ is measurable, being the projection onto the first coordinate of the Borel set $\{(x, y, C): x \in \Psi(y, C)\} \cap(X \times \mathcal{A})$.

We say that $(\nu, \pi)$ has indistinguishable infinite clusters if for every $\Gamma$-invariant Borel subset $\mathcal{A} \subset \mathfrak{C}_{\infty}^{\mathrm{cl}}$, the set of $x \in X$ for which some $(x, C) \in \mathcal{A}$ and some $(x, C) \in \mathfrak{C}_{\infty}^{\mathrm{cl}} \backslash \mathcal{A}$ has $\nu$-measure 0 . In

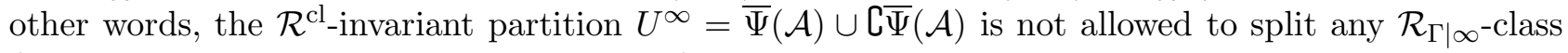
(up to a union of measure 0 of such classes). The following proposition, using this refined notion of indistinguishability, corrects [Gab05, Rem. 2.3]. 
Proposition 5 Let $\Gamma$ act ergodically on $(X, \nu)$ and $\pi: X \rightarrow\{0,1\}^{\mathrm{E}}$ be a $\Gamma$-equivariant Borel map such that $\nu\left(U^{\infty}\right) \neq 0$. Then $\mathcal{R}_{\mid \infty}^{\mathrm{cl}}$, the cluster equivalence relation restricted to its infinite locus $U^{\infty}$, is ergodic if and only if $(\nu, \pi)$ has indistinguishable infinite clusters.

Proof. Suppose that $\mathcal{R}_{\mid \infty}^{\mathrm{cl}}$ is ergodic. Then for every $\Gamma$-invariant Borel subset $\mathcal{A} \subset \mathfrak{C}_{\infty}^{\mathrm{cl}}$, its image $\bar{\Psi}(\mathcal{A})$ is a union of $\mathcal{R}_{\mid \infty}^{\text {cl }}$-classes, whence $\bar{\Psi}(\mathcal{A})$ or its complement $\complement \bar{\Psi}(\mathcal{A})$ in $U^{\infty}$ has measure 0 . In particular, the partition $U^{\infty}=\bar{\Psi}(\mathcal{A}) \cup \complement \bar{\Psi}(\mathcal{A})$ is trivial, whence $\nu$ has indistinguishable infinite clusters.

Conversely, suppose that $(\nu, \pi)$ has indistinguishable infinite clusters. An $\mathcal{R}_{\mid \infty}^{\mathrm{cl}}$-invariant partition $U^{\infty}=\mathcal{U} \cup \mathcal{C U}$ defines a partition $\mathfrak{C}_{\infty}^{\mathrm{cl}}=\mathcal{A} \cup \mathcal{C} \mathcal{A}$ according to whether $\Psi(x, C) \in \mathcal{U}$ or $\mathcal{C U}$. Then for $\nu$-almost every $x \in U^{\infty}$, all $\Psi(x, C)$ are in $\mathcal{U}$ or all are in its complement, i.e., the $\mathcal{R}_{\mid \infty}^{\text {cl }}$-subclasses into which the $\mathcal{R}_{\Gamma \mid \infty}$-class of $x$ splits all belong to one side. Since $\mathcal{R}_{\Gamma \mid \infty}$ is $\nu$-ergodic, this side has to be the same for almost every $x$. This means that the other side is a null set. This holds for any partition $\mathcal{U} \cup \mathcal{C U}$, whence $\mathcal{R}_{\mid \infty}^{\mathrm{cl}}$ is ergodic.

If $X$ has the form $X=\Omega \times Y$, then $\nu$ is called insertion tolerant (see [LS99]) if for each edge $e \in \mathrm{E}$, the map $\Pi_{e}: X \rightarrow X$ defined by $(\omega, y) \mapsto(\omega \cup\{e\}, y)$ quasi-preserves the measure, i.e., $\nu(A)>0$ implies $\nu\left(\Pi_{e}(A)\right)>0$ for every measurable subset $A \subseteq X$. Call a map $\pi: X \rightarrow \Omega$ increasing if $\pi(\omega, y) \supseteq \omega$ for all $\omega \in \Omega$. An action of $\Gamma$ on $\Omega \times Y$ is always assumed to act on the first coordinate in the usual way. A slight extension of [LS99, Th. 3.3, Rem. 3.4], proved in the same way, is the following:

Proposition 6 Assume that $\Gamma$ acts on $(\Omega \times Y, \nu)$ preserving the measure and $\pi: \Omega \times Y \rightarrow \Omega$ is an increasing $\Gamma$-equivariant Borel map with $\nu\left(U^{\infty}\right) \neq 0$. If $\nu$ is insertion tolerant, then $(\nu, \pi)$ has indistinguishable infinite clusters.

Proposition 7 If $\Gamma<\Lambda$, then there are $\Gamma$-equivariant isomorphisms $\left([0,1]^{\mathrm{E}}, \mu\right) \simeq\left([0,1]^{\Gamma}, \mu\right) \simeq$ $\left([0,1]^{\Lambda}, \mu\right)$ between the Bernoulli shift actions of $\Gamma$. In particular, the orbits of the Bernoulli shift $\Lambda$ action on $[0,1]^{\Lambda}$ are partitioned into subsets that are identified with the orbits of the standard Bernoulli shift $\Gamma$-action on $[0,1]^{\Gamma}$.

Proof. A countable set $\mathrm{E}$ on which $\Gamma$ acts freely may be decomposed by choosing a representative in each orbit so as to be identified with a disjoint union of $\Gamma$-copies, $\mathrm{E} \simeq \coprod_{J} \Gamma$, and to give $\Gamma$-equivariant identifications $[0,1]^{\mathrm{E}}=[0,1] \amalg^{\Gamma}=\left([0,1]^{J}\right)^{\Gamma}$.

The edge set $\mathrm{E} \simeq \amalg_{S} \Gamma$ of the Cayley graph of $\Gamma$, as well as $\Lambda \simeq \amalg_{I} \Gamma$ are such countable $\Gamma$-sets. Then isomorphisms of standard Borel probability spaces $([0,1], L e b) \simeq\left([0,1]^{S}, \otimes_{S} L e b\right) \simeq$ $\left([0,1]^{I}, \otimes_{I} L e b\right)$ induce $\Gamma$-equivariant isomorphisms of the Bernoulli shifts:

$$
\begin{array}{ccc}
{[0,1]^{\Gamma}} & \simeq\left([0,1]^{S}\right)^{\Gamma} & \simeq\left([0,1]^{I}\right)^{\Gamma} \\
\| & \| & \| \\
{[0,1]^{\Gamma}} & \simeq[0,1]^{\mathrm{E}} \simeq[0,1]^{\Lambda} .
\end{array}
$$


$-\mathrm{O}-$

A subgraph $\left(\mathrm{V}^{\prime}, \mathrm{E}^{\prime}\right)$ of a graph $(\mathrm{V}, \mathrm{E})$ is called spanning if $\mathrm{V}^{\prime}=\mathrm{V}$. A vertex $a$ in a graph is called a cutvertex if there are two other vertices in its component with the property that every path joining them passes through $a$. A block of a graph is a maximal connected subgraph that has no cutvertex. Every simple cycle of a graph is contained within one of its blocks.

Lemma 8 If all vertices of a block have finite degree and for each pair of vertices $(a, b)$ there are only finitely many distinct paths joining a to $b$, then the block is finite.

Proof. Suppose for a contradiction that the block is infinite. Then it contains a simple infinite path $P$ of vertices $a_{1}, a_{2}, \ldots$ By Menger's theorem, $a_{1}$ and $a_{n}$ belong to a simple cycle $C_{n}$ for each $n>1$. But this implies that there are infinitely many distinct paths joining $a_{1}$ to $a_{2}$ : Fix $n$ and let $a_{j}(2 \leq j \leq n)$ be the vertex on $P \cap C_{n}$ with minimal index $j$. We may assume that $C_{n}$ is oriented so that it visits $a_{n}$ before it visits $a_{j}$. Then simply follow $C_{n}$ from $a_{1}$ until $a_{j}$, and then follow $P$ to $a_{2}$.

Proposition 9 (For any Cayley graph) Let $X:=\Omega \times[0,1]^{\Gamma}$ and $\epsilon>0$. Let $\nu:=\mu_{\epsilon} \times \mu$. There is a $\Gamma$-equivariant Borel map $f: X \rightarrow \Omega$ such that $(\nu, f)$ has indistinguishable infinite clusters and for all sufficiently small $\epsilon$, the push-forward measure $f_{*}(\nu)$ of $\nu$ is supported on the set of spanning subgraphs of $\mathcal{G}$ each of whose components is a tree with infinitely many ends.

Proof. We may equivariantly identify $\left([0,1]^{\Gamma}, \mu\right)$ with $\left([0,1]^{\mathbb{N} \times \Gamma} \times[0,1]^{\mathbb{E}}, \mu \times \mu\right)$, so we identify $(X, \nu)$ with $\left(\Omega \times[0,1]^{\mathbb{N} \times \Gamma} \times[0,1]^{\mathrm{E}}, \mu_{\epsilon} \times \mu \times \mu\right)$. Fix an ordering of $S \amalg S^{-1}$; this determines an ordering of the edges incident to each vertex in $\mathcal{G}$, where we ignore edge orientations for the rest of this proof. With $d$ denoting the degree of $\mathcal{G}$, define the function $h(t):=\lceil d t\rceil$ for $t \in[0,1]$. Given a point $x=\left(\omega,(r(n, \gamma))_{n \in \mathbb{N}, \gamma \in \Gamma}, u\right) \in X$, construct the wired spanning forest $\mathcal{F}_{1}$ of $\mathcal{G}$ by using the cyclepopping algorithm of D. Wilson [Wil96, Sect. 3] as adapted in [BLPS01, Th. 5.1], also called there "Wilson's algorithm rooted at infinity", applied to the stacks where the $n$th edge in the stack under $\gamma$ is defined as the $h(r(n, \gamma))$ th edge incident to $\gamma$. The measure $\nu$ is insertion tolerant and the map $\pi: x \mapsto \omega \cup \mathcal{F}_{1}$ is increasing, whence by Proposition [6, the pair $(\nu, \pi)$ has indistinguishable infinite clusters. Notice that all clusters are infinite. Now use $u$ to construct the free minimal spanning forest $\mathcal{F}_{2}$ in each cluster of $\pi(x)$, that is, for every cycle $\Delta \subset \pi(x)$, delete the edge $e \in \Delta$ with maximum $u(e)$ in that cycle. The map $f$ is $f(x):=\mathcal{F}_{2}$.

Now the $\nu$-expected number of distinct simple paths in $\pi(x)$ that join any two vertices is finite (equation (13.7) of [BLPS01]) for all sufficiently small $\epsilon$. In particular, the number of such paths is finite $\nu$-a.s. By Lemma $\mathbb{8}$, this means that all blocks of $\pi(x)$ are finite, so that $\mathcal{F}_{2}$ is a spanning tree in each block. Therefore each component of $\mathcal{F}_{2}$ spans a component of $\pi(x)$. Thus, $\mathcal{F}_{2}$ determines the same cluster relation and so $(\nu, f)$ also has indistinguishable (infinite) clusters. Finally, the fact that the clusters of $\pi(x)$, and hence those of $\mathcal{F}_{2}$, have infinitely many ends follows, e.g., from BLPS01, Th. 13.7]. 
The cluster relation determined by $f$ of Proposition 9 is treeable and has cost larger than 1 by Gab00, Cor. IV.24 (2)], has finite cost (since the degree is bounded), and is ergodic by Proposition 5. Since we may equivariantly identify $\left(\Omega \times[0,1]^{\Gamma}, \mu_{\epsilon} \times \mu\right)$ with $\left([0,1]^{\Gamma}, \mu\right)$, we proved:

Proposition 10 For any Cayley graph of $\Gamma$, the Bernoulli action on $\left([0,1]^{\Gamma}, \mu\right)$ contains a treeable subrelation that is ergodic and has cost in the interval $(1, \infty)$.

At this point, we already have a reasonable answer to the analogue of von Neumann's problem, since "treeable relation" is the analogue of "free group" and cost $\mathcal{C}>1$ is, in the context of treeable relations, equivalent to non-amenability.

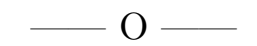

An alternative approach begins with a more explicit $f$ and a more common measure $f_{*}(\mu)$, namely, the Bernoulli measure $\mu_{p}$ on $\{0,1\}^{\mathrm{E}}$ for a certain parameter $p$, but requires us to choose a particular Cayley graph for $\Gamma$. It also requires us to obtain a treeable subrelation in a less explicit way. This is accomplished as follows.

Results of Häggström-Peres [HP99] imply that there are two critical values $0<p_{c} \leq p_{u} \leq 1$ such that

- (finite phase, $\left.p \in\left[0, p_{c}\right)\right) \mu_{p}$-a.s., the subgraph has only finite clusters;

- (non-uniqueness phase, $\left.p \in\left(p_{c}, p_{u}\right)\right) \mu_{p}$-a.s., infinitely many of the clusters of the subgraph are infinite, each one with infinitely many ends;

- (the uniqueness phase, $\left.p \in\left(p_{u}, 1\right]\right) \mu_{p}$-a.s., the subgraph has only one cluster that is infinite.

The situation for the critical values $p_{c}$ and $p_{u}$ themselves is far from clear. Benjamini and Schramm [BS96] conjectured that $p_{c} \neq p_{u}$ for every Cayley graph of a f.g. non-amenable group. The main result of PSN00] (Th. 1, p. 498) asserts that given a f.g. non-amenable group $\Gamma$, there is a finite set of generators such that the associated Cayley graph admits a non-trivial interval of non-uniqueness. Thus:

Proposition 11 (For particular Cayley graphs) There exists a Cayley graph of $\Gamma$ and a non-empty interval $\left(p_{c}, p_{u}\right)$ such that, for any $p \in\left(p_{c}, p_{u}\right)$, the Bernoulli measure $\mu_{p}$ on $\{0,1\}^{\mathrm{E}}$ is supported on the set of subgraphs admitting infinite components, each one with infinitely many ends.

Let $\pi:(X, \nu) \rightarrow\{0,1\}^{\mathrm{E}}$ denote either

(i) $f_{p}:\left([0,1]^{\mathrm{E}}, \mu\right) \rightarrow\{0,1\}^{\mathrm{E}}$ induced by the characteristic function $\chi_{[0, p]}:[0,1] \rightarrow\{0,1\}$ of $[0, p]$, or (ii) the identity map $\left(\{0,1\}^{\mathrm{E}}, \mu_{p}\right) \rightarrow\{0,1\}^{\mathrm{E}}$,

both with the natural Bernoulli $\Gamma$-action. Notice that the action is essentially free when $0<p<1$.

In case (ii), we have that $\left(\mu_{p}, \pi\right)$ has indistinguishable infinite clusters by [LS99, Th. 3.3]. Case (i) is essentially the same, but first we must identify $\left([0,1]^{\mathrm{E}}, \mu\right)$ equivariantly as $(\{0,1\} \times[0,1])^{\mathrm{E}}=$ $\{0,1\}^{\mathrm{E}} \times[0,1]^{\mathrm{E}}$ equipped with the product measure $\mu_{p} \times \mu$ in such a way that $f_{p}$ becomes the identity on the first coordinate. Then we have insertion tolerance and so, by [LS99, Rem. 3.4], indistinguishable infinite clusters. 
Hence, in both cases, for any $p$ given by Prop. 11, the locus $U^{\infty}$ of infinite classes of $\mathcal{R}^{\mathrm{cl}}$ is non-null and we have ergodicity of the restriction $\mathcal{R}_{\mid \infty}^{\mathrm{cl}}$ of $\mathcal{R}^{\mathrm{cl}}$ to $U^{\infty}$ by Proposition 5. We claim that its normalized cost (i.e., computed with respect to the normalized probability measure $\nu / \nu\left(U^{\infty}\right)$ on $U^{\infty}$ ) satisfies $1<\mathcal{C}\left(\mathcal{R}_{\mid \infty}^{\mathrm{cl}}\right)<\infty$. The finiteness of the cost is clear since $S$, the index set for $\Phi^{\mathrm{cl}}$, is finite. That it is strictly greater than 1 is a direct application of [Gab00, Cor. IV.24 (2)], since the graph $\Phi^{\mathrm{cl}}[x] \simeq \pi(x)_{\rho}$ associated with almost every $x \in U^{\infty}$ has at least 3 ends.

In order to extend $\mathcal{R}_{\mid \infty}^{\mathrm{cl}}$ to a subrelation of $\mathcal{R}_{\Gamma}$ defined on the whole of $X$, choose an enumeration $\left\{\gamma_{i}\right\}_{i \in \mathbb{N}}$ of $\Gamma$. For each $x \in X \backslash U^{\infty}$, let $\gamma_{x}$ be the first element $\gamma_{j} \in \Gamma$ such that $\gamma_{j} \cdot x \in U^{\infty}$. Then the smallest equivalence relation containing $\mathcal{R}_{\mid \infty}^{\mathrm{cl}}$ and the $\left(x, \gamma_{x} \cdot x\right)$ 's is a subrelation of $\mathcal{R}_{\Gamma}$, is ergodic, and has cost in $(1, \infty)$ by the induction formula of [Gab00, Prop. II.6]. We proved:

Proposition 12 For a Cayley graph and a p given by Proposition 11, the Bernoulli actions on both $\left([0,1]^{\mathrm{E}}, \mu\right)$ and $\left(\{0,1\}^{\mathrm{E}}, \mu_{p}\right)$ contain a subrelation that is ergodic and has cost in the open interval $(1, \infty)$.

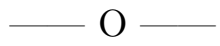

Proposition 13 If an equivalence relation $\mathcal{R}$ is ergodic and has cost in $(1, \infty)$, then it contains a treeable subrelation $\mathcal{S}_{1}$ that is ergodic and has cost in $(1, \infty)$.

Proof. This is ensured by a result proved independently by A. Kechris and B. Miller KM04, Lem. 28.11; 28.12] and by M. Pichot [Pic05, Cor. 40], through a process of erasing cycles from a graphing of $\mathcal{S}_{1}$ with finite cost that contains an ergodic global isomorphism.

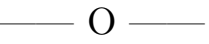

Proposition 14 If a treeable equivalence relation $\mathcal{S}_{1}$ is ergodic and has cost $>1$, then it contains a.e. a subrelation $\mathcal{S}_{2}$ that is generated by an ergodic free action of the free group $\mathbf{F}_{2}$.

Proof. If the cost of $\mathcal{S}_{1}$ is $>2$, this follows from a result of G. Hjorth Hjo06 (see also [KM04, Sect. 28]). Otherwise, one first considers the restriction of the treeable $\mathcal{S}_{1}$ to a small enough Borel subset $V$ : this increases the normalized cost by the induction formula of [Gab00, Prop. II.6 (2)] to get $\mathcal{C}\left(\mathcal{S}_{1} \mid V\right) \geq 2$. In fact, it follows from the proof of [KM04, Th. 28.3] that a treeable probability measure-preserving equivalence relation with cost $\geq 2$ contains a.e. an equivalence subrelation that is given by a free action of the free group $\mathbf{F}_{2}=\langle a, b\rangle$ in such a way that the generator a acts ergodically. By considering a subgroup of $\mathbf{F}_{2}$ generated by $a$ and some conjugates of $a$ of the form $b^{k} a b^{-k}$, one gets an ergodic treeable subrelation of $\mathcal{S}_{1} \mid V$ with a big enough normalized cost that, when extended to the whole of $X$ (by using partial isomorphisms of $\mathcal{S}_{1}$ ), it gets cost $\geq 2$ (by the induction back Gab00, Prop. II.6 (2)]) and of course remains ergodic. Another application of the above-italicized result gives the desired ergodic action of $\mathbf{F}_{2}$ on $X$. 
$-\mathrm{O}-$

The proof of Theorem 2 is now complete as a direct consequence of Propositions 12 (for the case $\left.X=\{0,1\}^{\mathrm{E}}\right)$, 13 and 14 .

In case $X=[0,1]^{\mathrm{E}}$ of Prop. 12, by using Prop. 7, we can see $\mathcal{S}_{2}$ (with $\mathcal{S}_{2} \subset \mathcal{S}_{1} \subset \mathcal{R}_{\Gamma}$ given by Prop. 14 and 13) as an equivalence subrelation of that given by the Bernoulli shift action of $\Lambda$. This finishes the proof of Theorem 11. Alternatively, we may use Prop. 10 and a similar argument to prove Theorem 1.

$$
\mathrm{O}-
$$

Proof of Cor 3. For any diffuse abelian subalgebra $A$ of $L(H)$, the von Neumann factor $L(H<\Lambda)=$ $L\left(\Lambda \ltimes \oplus_{\Lambda} H\right)$ contains the von Neumann algebra crossed product $\Lambda \ltimes \otimes_{\Lambda} A$, which is isomorphic with the group-measure-space factor $\Lambda \ltimes L^{\infty}\left([0,1]^{\Lambda}, \mu\right)$ associated with the Bernoulli shift. The corollary then follows from Th. 1 .

Proof of Cor. 因. If $\hat{K}$ is the dual group of $K$, then $L(H\langle\Gamma)$ contains $L(K \succ \Gamma)$, which is isomorphic with the group-measure-space factor $\Gamma \ltimes L^{\infty}\left(\hat{K}^{\Gamma}\right)$ associated with the Bernoulli shift of $\Gamma$ on $\hat{K}^{\Gamma}$, where the finite set $\hat{K} \simeq\{1,2, \ldots, k\}$ is equipped with the equiprobability measure $\nu$. The result is then obtained by taking the pull-back of the $\mathbf{F}_{2}$-action on $\prod_{1}^{n}\{0,1\}^{\Gamma}$, given in Th. 2 , by the $\Gamma$-equivariant Borel map $\hat{K}^{\Gamma} \rightarrow\left(\{0,1\}^{n}\right)^{\Gamma} \simeq \prod_{1}^{n}\{0,1\}^{\Gamma}$, sending $\otimes \nu$ to $\mu_{p}$, that extends a map $\{1,2, \ldots, k\} \rightarrow\{0,1\}^{n}$ (whose existence is ensured by the form of $\left.k=\beta^{n}\right)$.

It is likely that the free minimal spanning forest (FMSF) of a Cayley graph of $\Gamma$ would serve as the desired ergodic subrelation $\mathcal{S}_{1}$ of Prop. 13, but its indistinguishability, conjectured in [LPS06], is not known. Also, it is not known to have cost $>1$, but this is equivalent to $p_{c}<p_{u}$, which is conjectured to hold and which we know holds for some Cayley graph. See [LPS06 for information on the FMSF and [Tim06] for a weak form of indistinguishability.

A general question remains open:

Question: Does every probability-measure-preserving free ergodic action of a non-amenable countable group contain an ergodic subrelation generated by a free action of a non-cyclic free group? More generally: Does every standard countable probability-measure-preserving non-amenable ergodic equivalence relation contain a treeable non-amenable ergodic equivalence subrelation?

\section{References}

[BLPS01] Itai Benjamini, Russell Lyons, Yuval Peres, and Oded Schramm. Uniform spanning forests. Ann. Probab., 29(1):1-65, 2001.

[BS96] Itai Benjamini and Oded Schramm. Percolation beyond $\mathbf{Z}^{d}$, many questions and a few answers. Electron. Comm. Probab., 1:no. 8, 71-82 (electronic), 1996. 
[Gab00] Damien Gaboriau. Coût des relations d'équivalence et des groupes. Invent. Math., 139(1):41-98, 2000.

[Gab05] Damien Gaboriau. Invariant percolation and harmonic Dirichlet functions. Geom. Funct. Anal., 15(5):1004-1051, 2005.

[Hjo06] Greg Hjorth. A lemma for cost attained. Ann. Pure Appl. Logic, 143(1-3):87-102, 2006.

[HP99] Olle Häggström and Yuval Peres. Monotonicity of uniqueness for percolation on Cayley graphs: all infinite clusters are born simultaneously. Probab. Theory Related Fields, 113(2):273-285, 1999.

[KM04] Alexander S. Kechris and Benjamin D. Miller. Topics in Orbit Equivalence, volume 1852 of Lecture Notes in Mathematics. Springer-Verlag, Berlin, 2004.

[LPS06] Russell Lyons, Yuval Peres, and Oded Schramm. Minimal spanning forests. Ann. Probab., 34(5):1665-1692, 2006.

[LS99] Russell Lyons and Oded Schramm. Indistinguishability of percolation clusters. Ann. Probab., 27(4):1809-1836, 1999.

[Ol'80] Alexander Ju. Ol'šanskiu. On the question of the existence of an invariant mean on a group. Uspekhi Mat. Nauk, 35(4(214)):199-200, 1980.

[Pic05] Mikaël Pichot. Quasi-Périodicité et Théorie de la Mesure. Ph.D. Thesis, Ecole Normale Supérieure de Lyon, 2005.

[PSN00] Igor Pak and Tatiana Smirnova-Nagnibeda. On non-uniqueness of percolation on nonamenable Cayley graphs. C. R. Acad. Sci. Paris Sér. I Math., 330(6):495-500, 2000.

[Tim06] Ádám Timár. Ends in free minimal spanning forests. Ann. Probab., 34(3):865-869, 2006.

[vN29] John von Neumann. Zur allgemeinen Theorie des Maßes. Fund. Math., 13:73-116, 1929.

[Why99] Kevin Whyte. Amenability, bi-Lipschitz equivalence, and the von Neumann conjecture. Duke Math. J., 99(1):93-112, 1999.

[Wil96] David Bruce Wilson. Generating random spanning trees more quickly than the cover time. In Proceedings of the Twenty-eighth Annual ACM Symposium on the Theory of Computing (Philadelphia, PA, 1996), pages 296-303, New York, 1996. ACM.

Damien Gaboriau:

Unité de Mathématiques Pures et Appliquées

Université de Lyon, CNRS, Ens-Lyon 
46, allée d'Italie

69364 Lyon cedex 7, France

gaboriau@umpa.ens-lyon.fr

Russell Lyons:

Department of Mathematics

Indiana University Bloomington, IN 47405-5701

USA

rdlyons@indiana.edu 